\title{
CHARACTERIZATION OF SPATIAL VARIABILITY OF SPECTRAL IRRADIANCE IN TOBACCO CANOPY
}

\author{
LAO, C.L. ${ }^{1,4}-\mathrm{XU}, \mathrm{Z} . \mathrm{L}^{2}{ }^{2}-\mathrm{GUO}, \mathrm{Y}^{3}{ }^{3}-\mathrm{JIN}, \mathrm{Y}^{2}{ }^{2}-\mathrm{YANG}, \mathrm{Y} . \mathrm{H}^{2}{ }^{2}$ \\ ${ }^{1}$ China Agricultural University, Key Laboratory of Modern Precision Agriculture System \\ Integration Research, Ministry of Education, College of Information and Electrical Engineering \\ 17 Tsinghua East Road, Haidian district, Beijing, 100083, PR China \\ (phone: +86-10-6273-7898) \\ ${ }^{2}$ Yunnan Academy of Tobacco Agricultural Sciences \\ 14 Nanxiang Road, Gaoxin district, Yuxi, Yunnan, 653100, PR China \\ (phone: +86-877-207-5035) \\ ${ }^{3}$ China Agricultural University, College of Resources and Environment \\ 2 Yuanmingyuan West Road, Haidian district, Beijing, 100193, PR China \\ (phone: +86-10-6273-2959) \\ ${ }^{4}$ Boston University, Department of Geography and Environment \\ 675 Commonwealth Avenue, Boston, MA 02115, USA \\ (phone: +1-857-272-1297) \\ *Corresponding author \\ e-mail: toyyred@263.net \\ (Received $14^{\text {th }}$ Nov 2012; accepted $22^{\text {nd }}$ July 2014)
}

\begin{abstract}
Characterization of light micro-environment in plant canopy is vital in ecological and agricultural research. In this study, spatial heterogeneity of light micro-environment in tobacco canopies was characterized in biologically active radiation of blue, red, far-red, as well as photosynthetically active radiation (PAR) based on field measurements. The results revealed that spectral composition of light penetrating into these canopies varied significantly with both depth and horizontal locations, featured with abrupt drop in PAR and relatively mild decrease in far-red. The variations had good accordance of linear correlations between PAR, blue and red, whereas quadratic relations between far-red and other biologically active radiation in PAR. These spectral variations were manifested pronouncedly by R/FR (red to far-red ratio), a light signal perceived by plant organs for modulating their growth and development. The main contribution of this study is introducing a generic measurement method for charactering the spatial and spectral variation of light micro-environment within canopy. It is a basal and vital step to the profound study of the interaction between plants and their natural light micro-environment.
\end{abstract}

Keywords: plant; light environment; spatial heterogeneity; red to far-red ratio; biologically active radiation 


\section{Introduction}

The light micro-environment in plant canopy can vary dramatically with time and location not only in quantity but also in quality (spectral characteristics) (Grant, 1997; de Castro, 2000; Hertel et al., 2011). Plants had evolved themselves to possess a variety of photo-sensory systems with photoreceptors for perceiving tiny fluctuations in their light environment and responding with delicate modulation on their development and growth for capturing more energy and resources (Gilbert et al., 1995; Santner and Estelle, 2009; Stamm and Kumar, 2010). Phytochrome is the most well-characterized family of photoreceptors perceiving the ratio of red (R: 655-665 nm) to far-red (FR: 725-735 nm) and triggering shade avoidance response in the early phase of canopy development of higher plant (Ballaré et al., 1990; Aphalo et al., 1999; Smith, 2000). The variations in the blue (B: $400-500 \mathrm{~nm}$ ) domain can be sensed by cryptochrome which is another family of photoreceptors (Ahmad and Cashmore, 1993; Ballaré, 1999; Pierik et al., 2004; Sellaro et al., 2010).

Kasperbaue (1971) and Kasperbaue and Peaslee (1973) reported that in the environment-controlled laboratory, far-red irradiated tobacco plants developed longer internodes, longer, narrower and thinner leaves with fewer stomata and less chlorophyll, owing to the modulation of phytochrome. Two decades later, three phytochrome genes, designated Nt-PHYA1, Nt-PHYA2 and Nt-PHYB1, were isolated in tobacco plant. Their structures, expression patterns and levels were characterized in detail (Adam et al., 1997). The phytohormone signal network in photo -sensory system was being revealed (Achard et al., 2006; Santner and Estelle, 2009; Stamm and Kumar, 2010). Further studies on the interaction between tobacco plant and its natural light environment were expected.

Light environment in plant canopy has traditionally been quantified in the limited range of the photosynthetically active radiation (PAR, 400-700 nm) as photosynthetic photon flux density (PPFD). The spectral composition of light and its regulating function on plant's morphogenesis were ignored. This study aims to characterize the light in photoreceptor sensitive wavebands of B, R and FR as well as PAR. By measuring the above- and within-canopy spectral irradiance in the range of 350-1100 $\mathrm{nm}$, the spatial heterogeneity of irradiance in these biologically active radiations (Lee and Downum, 1991; Grant, 1997) and the spectral ratio of R/FR are examined.

\section{Materials and methods}

The field experiment was conducted at Yanhe Experiment Station $\left(24.23^{\circ} \mathrm{N}\right.$, $102.49^{\circ} \mathrm{E}$, Altitude: $1634 \mathrm{~m}$ ), Yuxi, Yunnan province in the southwest of China. Two tobacco (Nicotiana tabacum) cultivars, Y87 and K326, were employed in this study. Seedlings were transplanted to field plots (paddy soil) on May 11, 2011, in deep rooting way on northeast-southwest rows with $100 \mathrm{~cm}$ row distance and $50 \mathrm{~cm}$ plant-plant distance. The ridge was earthed up from $30 \mathrm{~cm}$ to $35 \mathrm{~cm}$ in height after planting. Fertilization, irrigation and pest control were performed according to the local standard cultivation of tobacco (Lei and Shi, 1999). 
Radiation measurements were performed in these two tobacco canopies (named as Y87P and K326P) from 11:00 to 12:00 on August 3, 2011. The plant height was about $100-120 \mathrm{~cm}$ and there was 20-25 leaves per plant. Leaf length ranged from $25-70 \mathrm{~cm}$ and maximum width from 10-30 cm. Leaf area indexes (LAI) were 2.61(Y87P) and $3.26(\mathrm{~K} 326 \mathrm{P})$, respectively. The sky was clear with casual broken cloud.

FieldSpec3 field spectroradiometer (ASD Inc., USA) was employed in this study and it was equipped with a Remote Cosine Receptor (RCR) (model A124505, ASD Inc.) with $180^{\circ}$ field of view. It had a detection range of $350-2500 \mathrm{~nm}$, with spectral resolution of $1.4 \mathrm{~nm}$ for the region $350-1000 \mathrm{~nm}$ and $2 \mathrm{~nm}$ for the region 1000-2500 $\mathrm{nm}$. Radiometric calibration of RCR has been performed by manufacturer for irradiance ( $\mathrm{W} \mathrm{m}^{-2} \mathrm{~nm}^{-1}$ ) calculation. The RCR is primarily designed for measuring hemispheric sky irradiance. In this study it was also used for measuring the irradiance within canopy in different depth and locations. To position the measurement precisely, we fixed the RCR facing upwards and horizontally on a 1-meter-length ruler which was bonded on a self-designed holder (Fig. 1). The holder was constructed with a vertical stick fixed on a stable base and a horizontal stick jointing on it. A horizontal plate was mounted on the horizontal stick for bonding the ruler. The horizontal stick can move freely in vertical direction along the vertical stick, and the horizontal plate can move in horizontal direction along the horizontal stick. The sticks were labeled evenly with $5 \mathrm{~cm}$ intervals in horizontal range of $60 \mathrm{~cm}$, and vertical range of $150 \mathrm{~cm}$.

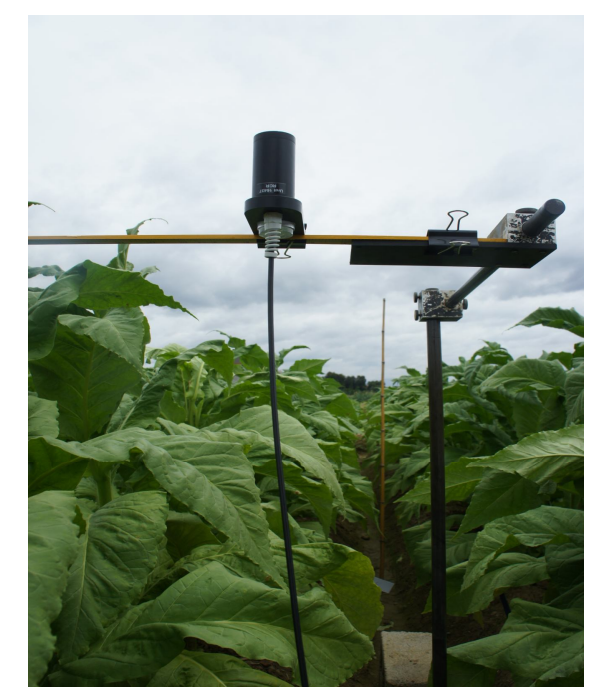

Figure 1. The picture of the self-designed holder for mounting the Remote Cosine Receptor and AccuPAR external sensor

Radiation measurement for each canopy was conducted in a sample plot including $2 \times 2$ (rows $\mathrm{x}$ plants in each row) plants at the center of the whole canopy. Fig. 2 presented the position of measuring points in one sample plot. We set the vertical measuring range $0-135 \mathrm{~cm}$ and horizontal (in the direction of cross rows) range $0-60 \mathrm{~cm}$ 
respectively. Since the maximum height of two canopies was $120 \mathrm{~cm}$ and their row distance was $100 \mathrm{~cm}$, the measurement can cover from above-canopy down to canopy bottom in depth, and cross a half of one sampling row in horizon.

For monitoring solar spectral irradiation above the canopy instantaneously with within-canopy measurements, it was preferable to make above and within canopy spectral irradiance measurements simultaneously. Considering we had only one spectroradiometer, we employed AccuPAR (model LP-80, Decagon Devices inc. USA) with an external sensor to monitor the photosynthetic photon flux density $\left(\mu \mathrm{mol} \mathrm{m} \mathrm{s}^{-2}\right.$ ) of solar irradiance instead of spectral irradiance when we used spectroradiometer for within-canopy measurement, based on the fact that the spectral composition of solar irradiation does not change significantly around midday in clear days (Jacovides et al., 2000). The external sensor was horizontally mounted on the top of the vertical stick of the holder.

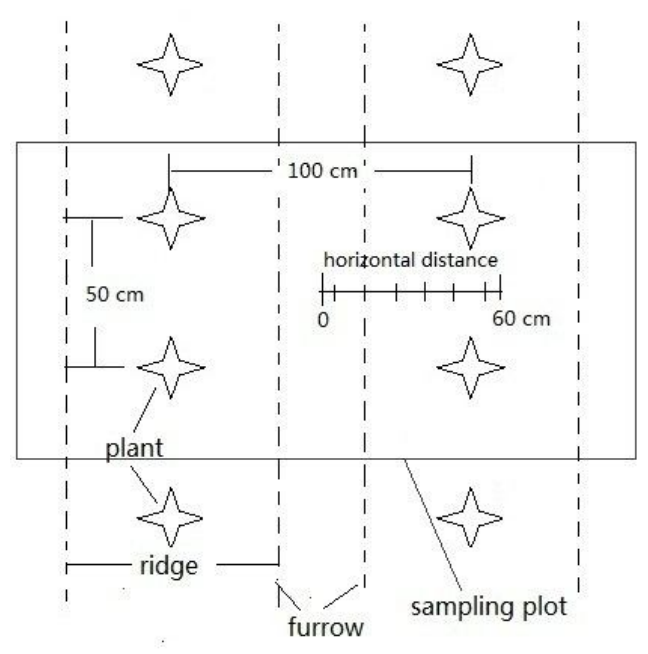

(a)

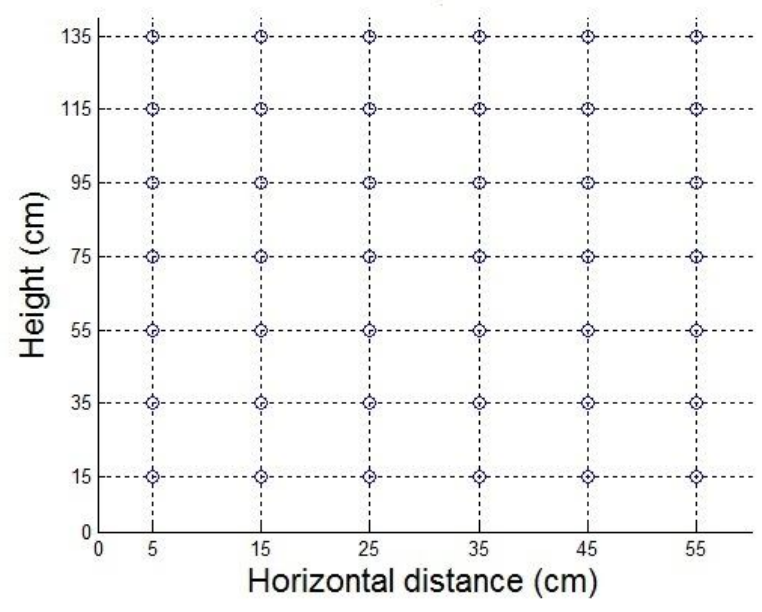

(b)

Figure 2. Scheme of measuring points in a sample plot: (a) The sampling scheme in horizontal direction, (b) The sampling scheme in both horizontal and vertical directions. The reference point $(0,0)$ is in the plane of ridge crest lines.

Before and after each within-canopy measurements, we took measurements of both spectral irradiance and corresponding PPFD for incident solar radiation instantaneously with interval of 1 minute. These observations were used to build the relationship between the above-canopy solar spectral irradiance with PPFD values.

For each measurement position, spectral irradiance was recorded by taking an average of 25 continuous scans from 350 to $1100 \mathrm{~nm}$ in $1 \mathrm{~nm}$ step. In the mean time, PAR readings above canopy were recorded. During the measurement, optical optimizing of spectrometer was performed when strong variation occurred, especially when the solar radiation increased dramatically, for getting response with high quality signal and avoiding signal saturation. 
Unary linear regression was used to build the relationship between the above-canopy solar spectral irradiance and PPFD values $\left(E_{P P F D}\right)$, with spectral irradiance $\left(E_{\lambda \_ \text {ac }}\right)$ of each wavelength as a dependent variable, and $E_{P P F D}$ as the independent variable:

$$
E_{\lambda_{-} a c}=C_{\lambda} E_{P P F D}
$$

The coefficient $C_{\lambda}$ is wavelength dependent, it was figured out with a least squares fit on observations, by minimizes the sum of the squares of the deviations between observed and modeled spectral irradiance as:

$$
\min =\sum_{n}\left(E_{\lambda_{-} a c}-C_{\lambda} E_{P P F D}\right)^{2}
$$

Where $\mathrm{n}$ is the number of observations.

For quantifying the percentage radiation penetrating into canopy, spectral transmittance $\left(\tau_{\lambda}\right)$ was defined as the ratio of within-canopy spectral irradiance $\left(E_{\lambda \_ \text {wc }}\right)$ to above-canopy spectral irradiance which is estimated from the measurement of PPFD by Eq. 1:

$$
\tau_{\lambda}=E_{\lambda_{-} w c} /\left(C_{\lambda} E_{P P F D}\right)
$$

Transmittance in biologically active radiation of PAR, B, R and FR were calculated using following equations:

$$
\begin{gathered}
\tau_{P A R}=\sum_{\lambda=400}^{700} E_{\lambda_{-} w c}\left(\sum_{\lambda=400}^{700} C_{\lambda} E_{P P F D}\right) \\
\tau_{B}=\sum_{\lambda=400}^{500} E_{\lambda_{-} w c} /\left(\sum_{\lambda=400}^{500} C_{\lambda} E_{P P F D}\right) \\
\tau_{R}=\sum_{\lambda=655}^{665} E_{\lambda_{-} w c}\left(\sum_{\lambda=655}^{665} C_{\lambda} E_{P P F D}\right) \\
\tau_{F R}=\sum_{\lambda=725}^{735} E_{\lambda_{-} w c} /\left(\sum_{\lambda=725}^{735} C_{\lambda} E_{P P F D}\right)
\end{gathered}
$$


$\mathrm{R} / \mathrm{FR}$ ratio was calculated with:

$$
R / F R=\sum_{\lambda=655}^{665} E_{\lambda} / \sum_{\lambda=725}^{735} E_{\lambda}
$$

\section{Results}

Above-canopy observations of solar spectral irradiance and corresponding PPFD were recorded before and after each of the two within-canopy measurements with 10 and 6 samples, respectively. The above-canopy observations for Y87P were grouped in group1 and those for K326P in group2. The measuring time for group1 was from 11:05 to 11:30 and from 11:35 to 12:00 for group2. It showed that the solar radiance spectrum had no significant variation in its shape within one-hour measurement, although its PPFD and magnitude changed pronouncedly with time (Fig.3).

The correlation analysis illustrated good linear correlation between solar irradiance at each wavelength with PPFD for each of the two groups, with correlation coefficients all around 0.9 (Fig.4a). In contrast, very poor correlation was found when pooling data from both groups (Fig.4a). So it is necessary to build local linear regressions for each group of observations. Fig. $4 b$ displayed the wavelength dependent linear regression coefficients $\mathrm{C}_{\lambda}$ (Eq. 1) of these two groups of observations calculated with Eq. 2.

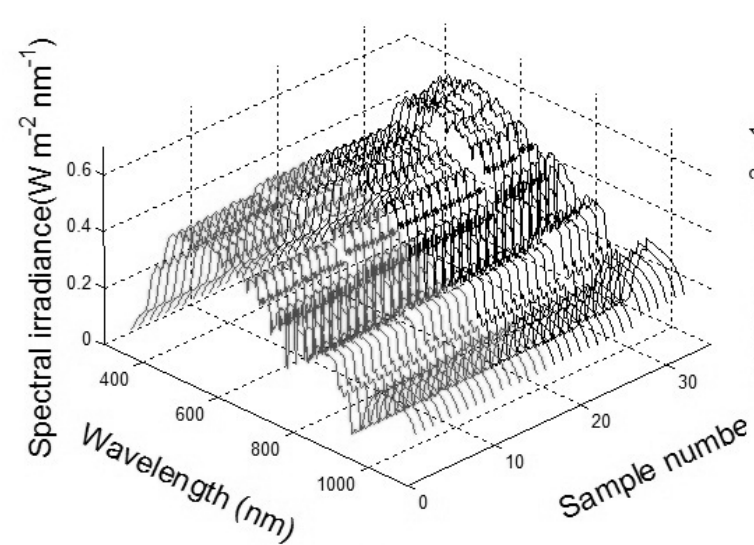

(a)

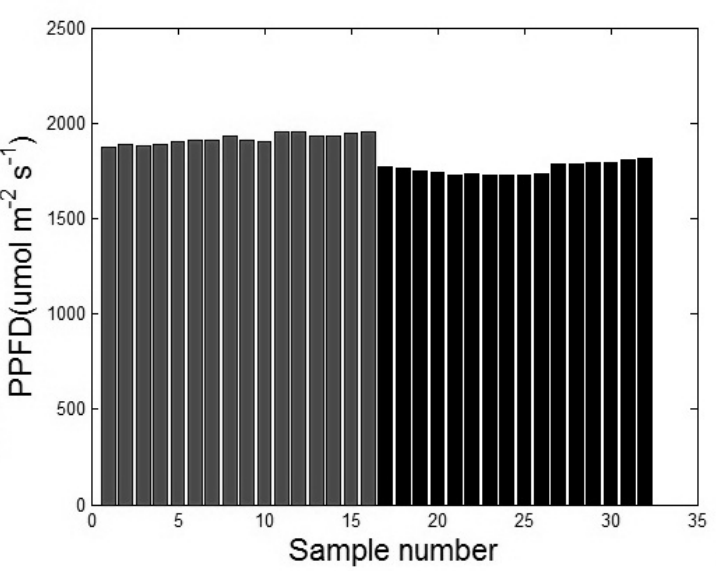

(b)

Figure 3. Observations (group1-gray, group2-black) of solar spectral irradiance (a) and corresponding PPFD (b) above Y87 and K326 canopies collected from 11:00 to 12:00 on August 3, 2011

Spectral irradiances were collected at all the measuring points including above and within canopies. The spectral transmittances at these measuring points were calculated with Eq.3, in which the wavelength dependent coefficients illustrated in Fig. $5 b$ were used to estimate solar spectral irradiances above canopy from PPFD measurements. 
Fig. 5 displayed the measured irradiances and calculated transmittances at four different depths which referring to above-canopy, upper, middle, and lower levels of K326P, respectively.

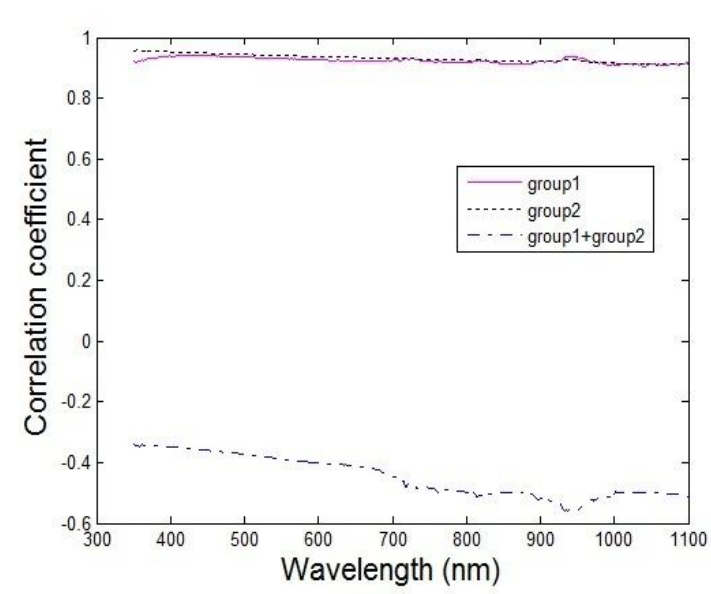

(a)

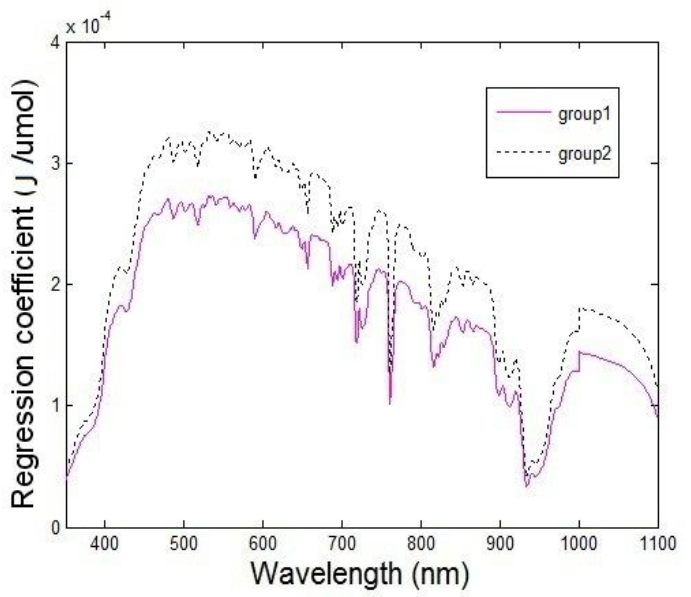

(b)

Figure 4. The relationship between above-canopy solar spectral irradiance and PPFD (a) Correlation coefficients (b) Regression coefficients

The measured spectral irradiances above canopy (Fig. $5 a$ left) manifested the same feature of the solar irradiation with relatively higher irradiance in PAR region and lower irradiance in far-red region. For upper part of canopy, the spectra collected near the center of sample row showed sharp drop in PAR while only small decrease in far-red region (Fig. $5 b$ left). With the increase in canopy depth, more measured points distant from center showed similar spectral variation (Fig. $5 c$-d left).

The transmittance spectra above canopy were constant with the same value of 1.0 (Fig.5a right). As they were calculated from PPFD measurements and spectral irradiance measurements, it illustrated the good agreement of solar spectral irradiance between the estimations from PPFD and measurements with spectroradiometer.

Spectral composition in the transmittance spectra within canopy (Fig.5b-d right) varied in large decrease of PAR and small decrease of FR with changes of depth and horizontal position. In the upper level of canopy, only a few of transmittances were lowered at the points near the center of row. With the increase in canopy depth (decrease of height), more points distant from row center had lower transmittances. This trend of variation kept good accordance with that of irradiance (Fig.5b-d left), and transmittance spectra manifested the spectral variation in irradiance distinctly. For instance, it is not easy to observe the variation of spectral composition in irradiance spectrum at horizontal distance of 5 and $15 \mathrm{~cm}$ with height of $95 \mathrm{~cm}$ (Fig. $5 \mathrm{~b}$ ), but it was clear in corresponding transmittance spectra that they were lower in PAR than FR. 

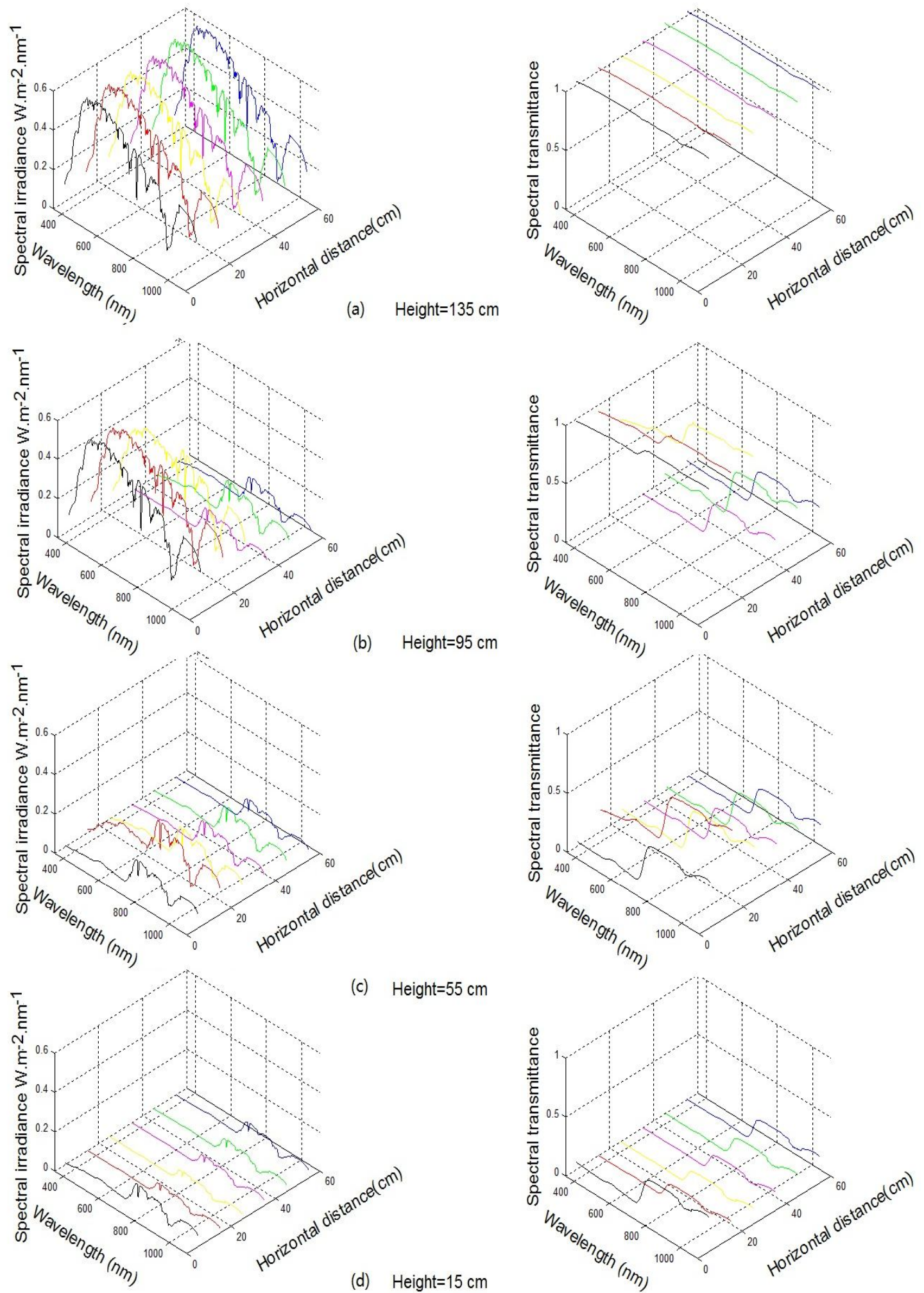

Figure 5. Spectral irradiance (left) and transmittance (right) at different height of K326 canopy 
The transmittances in PAR, B, R and FR were calculated for all measured points (Fig. 6). Transmittances in all these wavebands had a trend of decrease with the increase of depth on each vertical profile at the same horizontal position, with few exceptions which might be caused by the offset of measuring points for avoiding canopy disturbance. Abrupt decreases occurred in the profiles near the center of measuring row in a contrast to the relatively mild decrease in profiles near row's edge. This kind of contrast was more prominent in Y87P than in K326P. Fig.7 illustrated the average and deviation of transmittances in these four wavebands at different canopy heights. It is evident that Y87P had higher variation across horizontal planes than K326P.

Transmittances in PAR, B and R were very close at the same points except for FR which had much higher value when those in other wavebands were low. The scatter plots of transmittances for all the measurements between these wavebands demonstrated that transmittances in PAR, B and $\mathrm{R}$ had strong linear relations with coefficient of determination $\left(r^{2}\right)$ all greater than 0.99. Transmittance in FR also had positive correlation with PAR, B and R, but their relationship are more close to quadric rather than linear ones (Fig.8) and the coefficients of determination was higher for K326P $\left(r^{2}>0.99\right)$ than $Y 87 P\left(0.95>r^{2}>0.97\right)$.

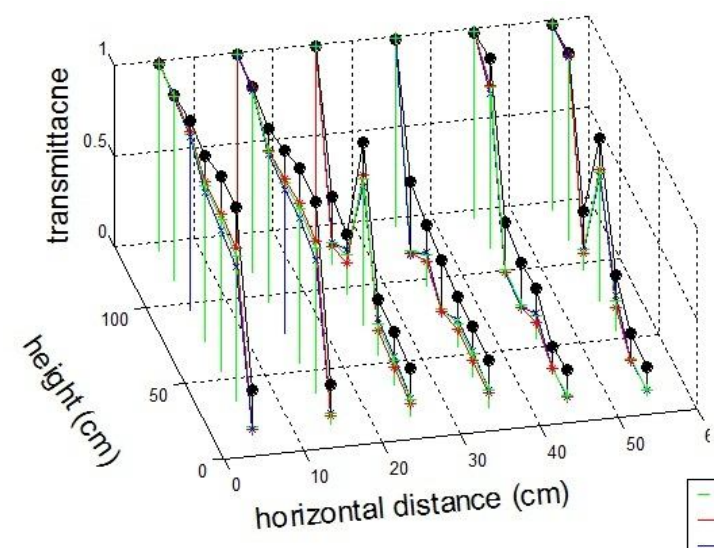

(a) Y87P

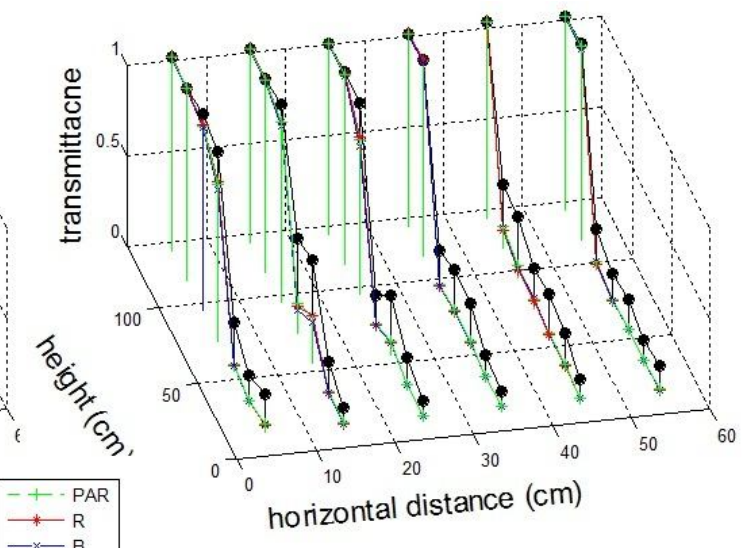

(b) K326P

Figure 6. Transmittances at different measured points in $Y 87$ and K326 canopies

$\mathrm{R} / \mathrm{FR}$ ratio at each measuring point was calculated for investigating its spatial variation within canopy (Fig.9). They had significant spatial variations within and between canopies. R/FR ratios were rather low at most points downwards from middle layers in K326P. In contrast, only a few points close to the bottom of Y87P canopy had low R/FR. The decrease of R/FR with increase of depth was milder in Y87P than that of K326P. 


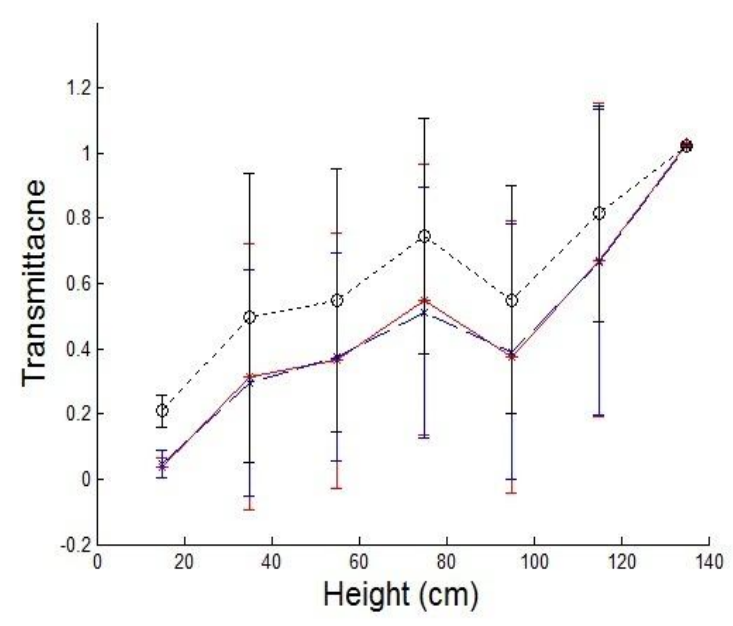

(a) Y87P

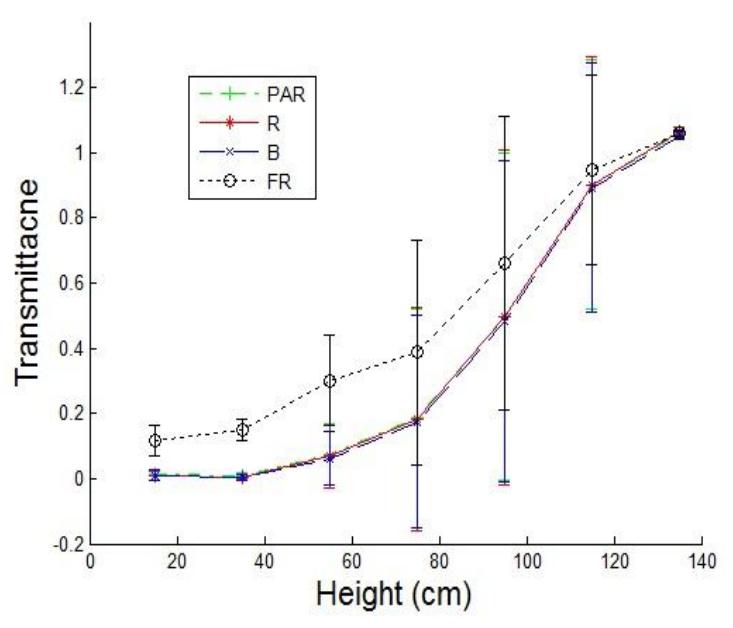

(b) K326P

Figure 7. Average transmittances in vertical profiles of $Y 87$ and K326 canopies

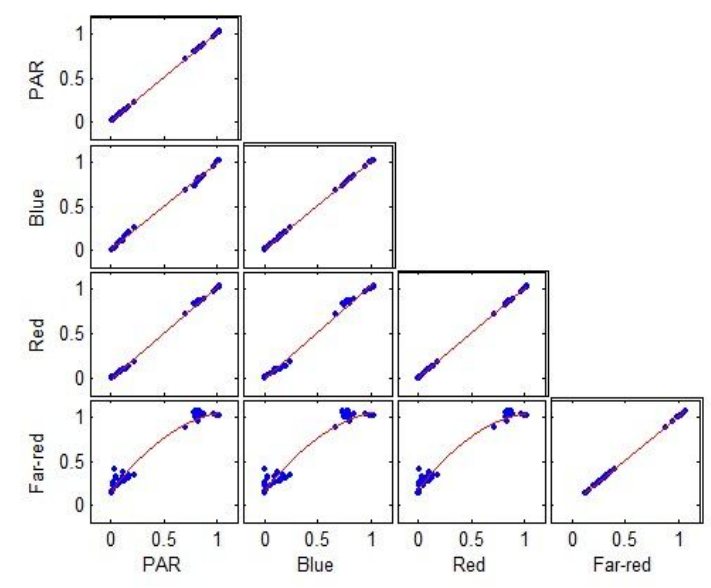

(a) Y87P

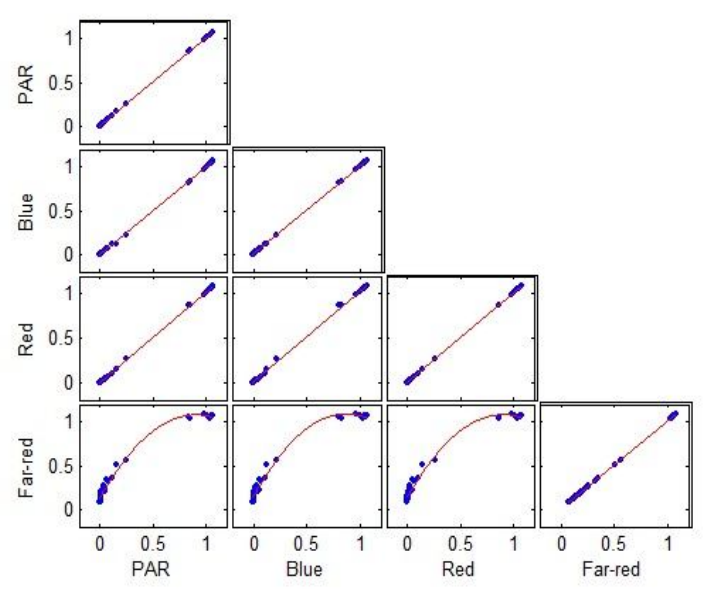

(b) K326P

Figure 8. Scatter plot of transmittance between PAR, Blue, Red and Far-red in Y87 (a) and K326 (b) canopies

\section{Discussion}

Although the spectral irradiance above canopy might vary with time according to the change of solar elevation and atmosphere conditions, its spectral composition did not have significant variation around midday under clear condition. As the spectral irradiance at each wavelength had good linear correlation with PPFD, it is acceptable to use measured PPFD for monitoring the solar irradiance and estimating solar spectral irradiance above canopy in clear midday. But we noticed that magnitude in spectral irradiances (Fig.3a) in group1 were lower than those in group2, which were in the 
reverse of PPFD values in these two groups (Fig.3b). The reason resulting in this system deviation was not clear yet. So it is recommended that their relationship should be examined frequently (at least with half an hour interval).

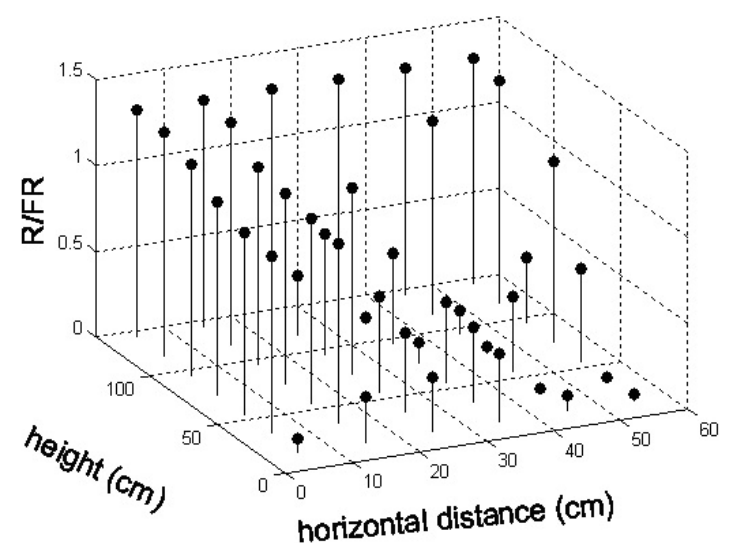

(a)

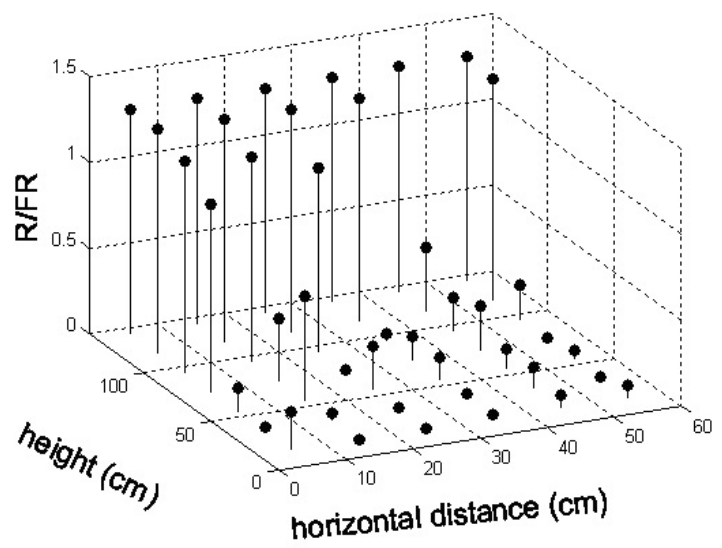

(b)

Figure 9. Spatial distribution of R/FR in $Y 87$ (a) and K326 (b) canopies

The measurements of within-canopy spectral irradiance demonstrated significant spatial and spectral variations in canopy light micro-environment, and their variations keep good accordance with spectral transmittance. As spectral transmittance in a medium is mostly determined by the property of medium, we could conclude that the variation of irradiance in spectrum composition within canopy was dominated by the property of canopy rather than solar spectral irradiation in clear midday. The spectral characteristic of lowest transmittance in blue and red waveband attributed to the selective absorption of blue and red light by chlorophyll in the leaves within canopy, and the spatial variation of spectral irradiance within canopy was affected by canopy architecture which was determined by spatial distribution of leaves (Panferov et al., 2001)

The decrease of transmittance in $\mathrm{B}, \mathrm{R}$ and PAR were all caused by selective absorption of chlorophyll in leaves (Walter-Shea and Norman, 1991). It made the variation of transmittance in PAR, B and R having good linear relations. High scattering and no significant absorbance of FR in leaves made the variation of FR quite different from those of PAR, R and $\mathrm{B}$ and kept more information of the spatial distribution of leaves. So it was reasonable to take the spatial variation of $\mathrm{R} / \mathrm{FR}$ as the indicator of spatial heterogeneity of canopy structure. The spatial distribution of R/FR in two canopies inferred that most places downward from middle in K326P were shaded by upper canopy apparatus. On the contrary, Y87P was much sparser in most part of the canopy except for the central part. It agreed to the fact that K326P had higher LAI than Y87P.

Quadric relationship was found between transmittances of FR with PAR, B and R in 
both Y87P and K326P, and it fit better for K32P which had high LAI. It might reveal some kind of relationship between quality and quantity of light within canopies. More theoretical and experimental investigations are needed for characterizing these relations.

In spite of the great achievements in the understanding of plant's photo-sensory system, most of the knowledge about the role played by various photoreceptor systems in plant-plant interactions was derived from physiological studies in which plant responses to specific light signals rather than actual plant neighbors in the field (Ballaré, 1999; Aphalo et al., 1999). Long-term field observation of canopy architecture and its light micro-environment in morphogenetic stage is necessary to fill this gap. Furthermore, orientation of the photoreceptorshould be taken into account since it has been revealed that in natural environment plants use the increase in horizontal FR to sense and respond to neighbors with increased elongation growth (Ballaré et al., 1990; Grant, 1997).

However, due to the constraint of experimental conditions, field measurement of spectral irradiance within canopy was limited to the hemispherical irradiance, and most of them were carried out in tree canopies (Turnball and Yates, 1993; de Castro, 2000; Leuchner, 2007; Hertel et al., 2011). It is always difficult to measure directional spectral irradiance on the surface of plant organisms within a field-grown crop canopy which has a complicated structure. Computer simulation of radiation transfer within three-dimensional canopy can be a good supplement tool (Panferov et al., 2001; Chelle and Andrieu 2007; Lao et al. 2010). But it should be based on the field measurement for providing primary experimental data and validating the results of simulation. Based on this study, we could build the relationship between field measurements and the simulations by three-dimensional radiative transfer model.

Acknowledgements. This research was supported by China National Tobacco Corporation Yunnan Province Company (2011YN08) and China National Tobacco Corporation (110201102008).

\section{REFERENCES}

[1] Achard, P., Cheng, H., de Grauwe, L., Decat, J., Schoutteten, H., Moritz, T., van der Straeten, D., Peng, J., Harberd N.P. (2006): Integration of plant responses to environmentally activated phytohormonal signals. - Science 311: 91-94.

[2] Ahmad, M., Cashmore, A.R. (1993): HY4 gene of A. thaliana encodes a protein with characteristics of a blue-light photoreceptor. - Nature 366: 162-166.

[3] Adam, E., Kozma-Bognar, L., Schafer, E., Nagy, F. (1997): Tobacco phytochromes: genes, structure and expression. - Plant, Cell and Environment 20: 678-684.

[4] Aphalo, P.J., Ballaré, C.L., Scopel, A.L. (1999): Plant-plant signaling, the shade avoidance response and competition. - Journal of Experimental Botany 50: 1629-1634.

[5] Ballaré, C.L., Scopel, A.L., Sánchez, R.A. (1990): Far-red radiation reflected from adjacent leaves: an early signal of competition in plant canopies. - Science 247: 329-331. 
[6] Ballaré, C.L. (1999): Keeping up with the neighbours: phytochrome sensing and other signalling mechanisms. - Trends in Plant Science 4: 97-102.

[7] Chelle, M., Andrieu, B. (2007): Modelling the Light Environment of Virtual Crop Canopies. - In: Vos, J., Marcelis, L.F.M., de Visser, P.H.B., Struik, P.C., Evers, J.B., (ed.) Functional-Structural Plant Modelling in Crop Production. Springer, Netherlands.

[8] de Castro, F. (2000): Light spectral composition in a tropical forest: measurements and model. - Tree Physiology 20: 49-56.

[9] Gilbert, I.R., Seavers, G.P., Jarvis, P.G., Smith, H. (1995): Photomorphogenesis and canopy dynamics. phytochrome-mediated proximity perception accounts for the growth dynamics of canopies of Populus trichocarpa $\times$ deltoides 'Beupré'. - Plant, Cell and Environment 18: 475-497.

[10] Grant, R.H. (1997). Partitioning of biologically active radiation in plant canopies. International Journal of Biometeorology 40: 26-40.

[11] Hertel, C., Leuchner, M., Menzel, A. (2011): Vertical variability of spectral ratios in a mature mixed forest stand. - Agricultural and Forest Meteorology 151: 1096-1105.

[12] Kasperbaue, M.J. (1971): Spectral distribution of light in a tobacco canopy and effects of end-of-day light quality on growth and gevelopment. - Plant Physiology 47: 775-778.

[13] Kasperbaue, M.J. and Peaslee, D.E. (1973): Morphology and photosynthetic efficiency of tobacco leaves that received end-of-day red or far red light during development. - Plant Physiology 52: 440-442.

[14] Jacovides, C.P., Steven, M.D., Asimakopoulos, D.N. (2000): Solar spectral irradiance under clear skies around a major metropolitan area. - Journal of Applied Meteorology 39: 917-930.

[15] Lao, C.L., Guo, Y., Li, B.G. (2010): Simulating the Distribution of R/FR in Maize Canopies with Monte Carlo Ray Tracing Approach. - In: Li, B.G., Jaeger, M., Guo, Y., (ed.) Plant Growth Modeling, Simulation, Visualization and Applications, Proceedings PMA09, IEEE CPS, Los Alamitos.

[16] Lee, D.W., Downum, K.R. (1991): The spectral distribution of biologically active solar radiation at Miami, Florida, USA. - International Journal of Biometeorology 35: 48-54.

[17] Lei, Y.H., Shi, Y.C. (1999): High Quality Tobacco Cultivation Techniques Mode. Yunnan Science and Technology Press, Kunming. (in Chinese).

[18] Leuchner, M., Menzel, A., Werner, H. (2007): Quantifying the relationship between light quality and light availability at different phenological stages within a mature mixed forest. - Agricultural and Forest meteorology 142(1): 35-44.

[19] Panferov, O., Knyazikhin, Y., Myneni, R.B., Szarzynski, J., Engwald, S., Schnitzler, K.G., Gravenhorst, G. (2001): The role of canopy structure in the spectral variation of transmission and absorption of solar radiationin vegetation canopies. - IEEE Trans. Geosci. Remote Sensing 39: 241-253.

[20] Pierik, R., Whitelam, G.C., Voesenek, L.A.C.J., de Kroon, H., Visser, E.J. W. (2004): Canopy studies on ethylene-insensitive tobacco identify ethylene as a novel element in blue light and plant-plant signaling. - Plant Journal 38: 310-319. 
[21] Santner, A., Estelle, M. (2009). Recent advances and emerging trends in plant hormone signalling. - Nature 459: 1071-1078.

[22] Smith, H. (2000): Phytochromes and light signal perception by plants -an emerging synthesis. - Nature 407: 585-591.

[23] Stamm, P., Kumar, P.P. (2010): The phytohormone signal network regulating elongation growth during shade avoidance. - Journal of Experimental Botany 61(11): 2889-2903.

[24] Sellaro, R., Crepy, M., Trupkin, S.A., Karayekov, E., Buchovsky, A.S., Rossi, C., Casal, J.J. (2010): Cryptochrome as a sensor of the blue/green ratio of natural radiation in arabidopsis. - Plant Physiology 154: 401-409.

[25] Turnball, M.H., Yates, D.J. (1993): Seasonal variation in the red/far-red ratio and photon flux density in an Australian sub-tropical rainforest. - Agricultural and Forest Meteorology 64: 111-127.

[26] Walter-Shea, E.A., Norman, J.M. (1991): Leaf optical properties. - In: Photon-vegetation interactions. Springer, Heidelberg. 